Note

\title{
Determination of Rodenticide Tetramethylenedisulfotetramine (Tetramine) in Processed Foods by Gas Chromatography-Tandem Mass Spectrometry
}

\author{
Shizuka Saito-Shida*, Satoru Nemoto, Reiko Teshima and Hiroshi AkiYama \\ National Institute of Health Sciences: 1-18-1 Kamiyoga, Setagaya-ku, Tokyo 158-8501, Japan; \\ * Corresponding author
}

\begin{abstract}
A GC-MS/MS method for determination of the rodenticide tetramethylenedisulfotetramine was developed. Tetramethylenedisulfotetramine was extracted from the sample with ethyl acetate in the presence of anhydrous sodium sulfate. Then, an aliquot of the extract was evaporated under vacuum, followed by acetonitrile/hexane partitioning, and cleanup with a tandem graphitized carbon/primary secondary amine (PSA) column, prior to GC-MS/MS analysis. The recoveries from 10 processed foods, all of which were fortified at $0.1 \mathrm{mg} / \mathrm{kg}$, were in the range of $85-96 \%$, and the relative standard deviations were less than $7 \%$. The proposed method effectively removed co-extracted matrix components, and matrix effects were negligible in the GC-MS/MS analysis. In addition, no interfering peaks were found in the chromatograms of the blank samples at the retention time of tetramethylenedisulfotetramine, indicating that the method is highly selective. Overall results suggest that the proposed method is suitable for determining tetramethylenedisulfotetramine contained in processed foods.
\end{abstract}

(Received February 16, 2016)

Key words: tetramethylenedisulfotetramine; rodenticide; GC-MS/MS; processed food

\section{Introduction}

Tetramethylenedisulfotetramine (2,6-dithia-1,3,5,7tetraazatricyclo[3.3.1.1 $\left.1^{3,7}\right]$ decane 2,2,6,6-tetraoxide) (Fig. 1 ), also known as tetramine, is a highly toxic rodenticide that was once used worldwide. It is an odorless, tasteless, white crystalline powder that easily dissolves in water and acts by binding noncompetitively and irreversibly to $\gamma$-aminobutyric acid (GABA) receptors on neuronal cell membranes and by blocking the chloride channel ${ }^{1-3)}$. Toxicity data for tetramine are limited; the median lethal dose $\left(\mathrm{LD}_{50}\right)$ is estimated as $0.1-0.3 \mathrm{mg} / \mathrm{kg}$ for mammals, and a dose of $7-10 \mathrm{mg}$ is considered to be lethal for humans ${ }^{1)-3}$. No ARfD (acute reference dose) value is available for tetramine. Tetramine has been banned in China since 1984 and has never been registered in Japan or the United State. However, from 1977 to 2002, many accidental and intentional poisonings occurred in China, resulting in hundreds of human fatalities $^{1-3)}$. Tetramine is not absorbed through the skin, and the most common route of exposure is ingestion of accidentally or intentionally contaminated foods. Thus, the development of a reliable method for the determination of tetramine in various foods is important to monitor the tetramine content in foods when accidental and intentional poisonings occur.

*shizsaito@nihs.go.jp
Several methods for the quantitative analysis of tetramine in biological samples, such as urine ${ }^{4), 5)}$ and blood $^{4), 6)}$, using gas chromatography (GC) coupled with nitrogen phosphorus detection (NPD) or mass spectrometry (MS) have been reported. However, only a few methods have been reported for the analysis of tetramine in foods ${ }^{7)}$ and beverages ${ }^{8)}$.

The aim of this study was to develop a reliable method for the determination of tetramine in foods. Although tetramine contamination can occur in both fresh and processed foods, we have targeted processed foods, which are complex mixtures of various food components of animal and plant origin. The sample preparation procedure was carefully optimized, and the proposed method was applied to 10 processed foods (broiled eel, butter, cheese, Chinese dumpling, hamburger, pizza, instant noodles, kimchi, red wine, and retort-pouch curry).

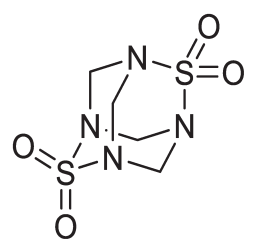

Fig. 1. Chemical structure of tetramethylenedisulfotetramine (tetramine) 


\section{Materials and Methods}

\section{Reagents and materials}

Pesticide-analysis-grade acetonitrile, ethyl acetate, and $n$-hexane were obtained from Kanto Chemical (Tokyo, Japan). Diatomaceous earth (Celite ${ }^{\circledR}$, No. 545) and pesticide-analysis-grade anhydrous sodium sulfate were purchased from Wako Pure Chemical Industries, Ltd. (Osaka, Japan).

The tetramine standard $(100.0 \pm 0.5 \mu \mathrm{g} / \mathrm{mL}$ in acetonitrile, 99.4\%) was purchased from Sigma-Aldrich (St. Louis, MO, USA). Stock standard solutions were prepared by dilution with acetonitrile. Calibration standard solutions were freshly prepared by diluting the stock standard solutions with acetonitrile.

The tandem graphitized carbon/primary secondary amine (PSA) column (InertSep GC/PSA, $500 \mathrm{mg} / 500 \mathrm{mg}$ ) was obtained from GL Sciences (Tokyo, Japan). PP centrifuge tubes $(15 \mathrm{~mL})$ were purchased from AGC Techno Glass (Shizuoka, Japan).

Broiled eel, butter, cheese, frozen Chinese dumpling, frozen hamburger, frozen pizza, instant noodles, kimchi, red wine, and retort-pouch curry were purchased from a market in Tokyo (Japan), and homogenized using a food processor.

\section{Instrumentation}

A Trace 1310 (ThermoFisher Scientific, MA, USA) gas chromatograph coupled to a TSQ 8000 mass spectrometer (ThermoFisher Scientific) and TriPlus RSH autosampler (ThermoFisher Scientific) was used, with the following operating conditions: column, DB-5 ms column (30 m length, $0.25 \mathrm{~mm}$ i.d., $0.25 \mu \mathrm{m}$ particle size; Agilent) with a guard column (2 m length, $0.25 \mathrm{~mm}$ i.d.; Agilent); column temperature, $50^{\circ} \mathrm{C} \quad(1 \mathrm{~min}) \rightarrow 25^{\circ} \mathrm{C} / \mathrm{min} \rightarrow 125^{\circ} \mathrm{C}$ $(0 \mathrm{~min}) \rightarrow 10^{\circ} \mathrm{C} / \mathrm{min} \rightarrow 300^{\circ} \mathrm{C}(8.5 \mathrm{~min})$; carrier gas, helium; flow rate, $1 \mathrm{~mL} / \mathrm{min}$; injection volume, $1 \mu \mathrm{L}$; ionization mode, electron ionization (EI) mode, ion source temperature, $260^{\circ} \mathrm{C}$; injection port temperature, $260^{\circ} \mathrm{C}$; transfer line temperature, $280^{\circ} \mathrm{C}$; retention time, $11.9 \mathrm{~min}$; selected reaction monitoring (SRM) transitions for quantification $m / z 240.0 \rightarrow 212.0$ (collision energy, $12 \mathrm{eV}$ ), and for confirmation $m / z 212.0 \rightarrow 132.1$ (collision energy, $8 \mathrm{eV}$ ).

A homogenizer (Polytron PT 10-35 GT; Kinematica, Lucerne, Switzerland), food processer (Grindomix GM 200; Retsch, Haan, Germany), rotary evaporator (N1000/NVC-2100; Tokyo Rikakikai, Tokyo, Japan), electric shaker (SR-2w; Taitec, Saitama, Japan), centrifuge (Centrifuge 8100; Kubota, Tokyo, Japan), and vacuum pump (APN-215 MV-1; Iwaki, Tokyo, Japan) connected to a Kiriyama funnel (Kiriyama Glass Works, Tokyo, Japan) containing filter paper No. 5B were also used.

\section{Sample preparation}

A $10.0 \mathrm{~g}$ portion of sample was weighed into a $350-\mathrm{mL}$ glass tube and then homogenized with $50 \mathrm{~mL}$ of ethyl acetate in the presence of $50 \mathrm{~g}$ of anhydrous sodium sulfate. The homogenate was filtered under vacuum through Celite, and the residue was washed twice with
$15 \mathrm{~mL}$ of ethyl acetate, and vacuum filtered. The combined filtrate was made up to $100 \mathrm{~mL}$ with ethyl acetate.

A $10 \mathrm{~mL}$ aliquot of the extract was concentrated to approximately $1 \mathrm{~mL}$ with a rotary evaporator below $40^{\circ} \mathrm{C}$ and evaporated to dryness under a stream of nitrogen. Then, the residue was transferred to a $15 \mathrm{~mL} P P$ centrifuge tube with $5 \mathrm{~mL}$ of $n$-hexane and $5 \mathrm{~mL}$ of acetonitrile saturated with $n$-hexane, shaken vigorously for $5 \mathrm{~min}$, and centrifuged at 3,000 rpm for $5 \mathrm{~min}$. The upper hexane layer was removed, and the resultant acetonitrile layer was loaded on a tandem graphitized carbon/ PSA (500 mg/500 mg) column, which was preconditioned with $10 \mathrm{~mL}$ of acetonitrile, and eluted with an additional $4 \mathrm{~mL}$ of acetonitrile. The combined eluate was made up to $10 \mathrm{~mL}$ with acetonitrile and used as test solution.

\section{Recovery tests}

Recovery tests were performed for broiled eel, butter, cheese, Chinese dumpling, hamburger, pizza, instant noodles, kimchi, red wine, and retort-pouch curry, with a spiking level of $0.1 \mathrm{mg} / \mathrm{kg}$, and with 5 replicates for all matrices. A $1 \mathrm{~mL}$ aliquot of a working standard solution $(1 \mu \mathrm{g} / \mathrm{mL})$ was added to the samples and allowed to stand for $30 \mathrm{~min}$ before extraction. The recoveries were calculated based on the peak area of tetramine, and they were quantified by external calibration using solvent standards $(0.0025,0.005,0.0075,0.01,0.0125$, and $0.015 \mu \mathrm{g} / \mathrm{mL})$.

\section{Evaluation of matrix effect}

Matrix-matched standards were prepared as follows: blank extract solution $(100 \mu \mathrm{L})$ was evaporated to dryness under a stream of nitrogen, and the residue was redissolved in $100 \mu \mathrm{L}$ of a $0.01 \mu \mathrm{g} / \mathrm{mL}$ standard in acetonitrile. The matrix effect was evaluated by comparing the peak area of tetramine in pure solvent with the peak area in matrix.

\section{Cleanup studies}

The elution profile of tetramine in a tandem graphitized carbon/PSA column was investigated as follows: $100 \mu \mathrm{L}$ of a $1 \mu \mathrm{g} / \mathrm{mL}$ tetramine standard in acetonitrile was evaporated to dryness under a stream of nitrogen, and the residue was dissolved in $5 \mathrm{~mL}$ of the acetonitrile layer after acetonitrile/hexane partitioning. The solution was loaded on a tandem graphitized carbon/PSA column, which was preconditioned with $10 \mathrm{~mL}$ of acetonitrile, and eluted (fraction 1). Then, the column was further eluted with $10 \mathrm{~mL}$ of acetonitrile (fraction 2, 0-3 mL; fraction 3, 3-5 mL; fraction 4, 5-10 mL). Each fraction was concentrated separately to approximately $1 \mathrm{~mL}$ with a rotary evaporator below $40^{\circ} \mathrm{C}$ and evaporated to dryness under a stream of nitrogen. The residues were dissolved in $1 \mathrm{~mL}$ of acetonitrile, and were examined by GC-MS/MS. 


\section{Results and Discussion}

\section{GC-MS/MS conditions}

In the mass spectrum of tetramine, a molecular ion $\left(\mathrm{M}^{+}\right)$ of $\mathrm{m} / z 240$ and its fragment ion of $\mathrm{m} / z 212$ were observed as the major ions. SRM transitions were optimized by varying the collision energy in the range of $6-14 \mathrm{eV}$ in $2 \mathrm{eV}$ steps. As a result, the precursor ion of $\mathrm{m} / z 240$ yielded two major product ions, $\mathrm{m} / z 212$ and 121 , while the precursor ion of $m / z 212$ yielded product ions of $\mathrm{m} / z 132$ and 92 . The two most intense ions, $\mathrm{m} / z$ $240 \rightarrow 212$ (collision energy $12 \mathrm{eV}$ ) and $\mathrm{m} / z \quad 212 \rightarrow 132$ (collision energy $8 \mathrm{eV}$ ), were selected as quantification and confirmation ions, respectively. The calibration curve was linear, with a correlation coefficient $(r)$ of 0.9996 in the concentration range of $0.001-0.2 \mu \mathrm{g} / \mathrm{mL}$

\section{Sample preparation}

In accordance with the "Rapid analytical method for pesticides containing high levels in processed foods" (Office Memorandum, Standards and Evaluation Division, Department of Food Safety, Ministry of Health, Labour and Welfare, Japan, March 26, 2013) ${ }^{* 1}$, tetramethylenedisulfotetramine was extracted from samples with ethyl acetate in the presence of anhydrous sodium sulfate.

Since some processed foods contain large amounts of lipids, we considered that a procedure to remove low polarity lipids from the extracts would be necessary. Therefore, acetonitrile/hexane partitioning was investigated in order to remove lipids from food samples. The extraction of tetramine from $n$-hexane with acetonitrile resulted in high recovery. The volume of $n$-hexane and acetonitrile required for partitioning was then optimized using extracts of various foods. The partitioning of $1.0 \mathrm{~g}$ butter extract, which contains a large amount of lipids, required $5 \mathrm{~mL}$ each of $n$-hexane and acetonitrile saturated with $n$-hexane. Therefore, an acetonitrile/hexane partitioning step, using $5 \mathrm{~mL}$ each of $n$-hexane and acetonitrile saturated with $n$-hexane, was added before the column cleanup step, and low polarity matrix components were effectively removed.

To remove food pigments and acidic matrix components, a tandem graphitized carbon/PSA column was then selected to further clean the samples. Table 1 shows the recovery of tetramine from the tandem graphitized carbon/PSA column. In order to speed up the procedure, the acetonitrile layer obtained in the acetonitrile/hexane partitioning step was directly loaded onto the tandem graphitized carbon/PSA column and passed through with an additional $3 \mathrm{~mL}$ of acetonitrile, which gave satisfactory recovery. Since the volume of acetonitrile required for eluting tetramine from the column may vary depending on foods, $4 \mathrm{~mL}$ of acetonitrile was used to elute tetramine from the column. The volume of the resultant eluate was accurately made up to $10 \mathrm{~mL}$, and this was used as a test solution, which was directly

\footnotetext{
*1 http://www.mhlw.go.jp/topics/bukyoku/iyaku/syoku-anzen/ zanryu3/dl/h25_0326-1.pdf
}

Table 1. Recovery of tetramine from the tandem graphitized carbon/PSA column

\begin{tabular}{ccccc}
\hline \hline \multicolumn{4}{c}{ Recovery (\%) } & \\
\hline \multirow{2}{*}{ Acetonitrile layer } & \multicolumn{3}{c}{ Additional acetonitrile } & \multirow{2}{*}{ Total } \\
\cline { 2 - 4 } & $0-3 \mathrm{~mL}$ & $3-5 \mathrm{~mL}$ & $5-10 \mathrm{~mL}$ & \\
\hline 48 & 53 & $<1$ & $<1$ & 101 \\
\hline
\end{tabular}

${ }^{a}$ Acetonitrile layer $(5 \mathrm{~mL})$ after acetonitrile/hexane partitioning.

Table 2. Recoveries of tetramine from processed foods

\begin{tabular}{lcc}
\hline \hline Sample & Recovery (\%) & RSD (\%) \\
\hline Broiled eel & 85 & 5 \\
Butter & 94 & 1 \\
Cheese & 91 & 4 \\
Chinese dumpling & 96 & 3 \\
Hamburger & 94 & 3 \\
Pizza & 92 & 6 \\
Instant noodles & 89 & 5 \\
Kimchi & 96 & 5 \\
Red wine & 93 & 5 \\
Retort-pouch curry & 93 & 7 \\
\hline
\end{tabular}

injected into the GC-MS/MS. To avoid matrix effects as much as possible, the dilution factor was set to 10 . The optimized sample cleanup procedure effectively removed interfering components and the cleaned-up sample was suitable for GC-MS/MS analysis.

\section{Recovery tests}

The recovery tests were performed using 10 processed foods, i.e., broiled eel, butter, cheese, Chinese dumpling, hamburger, pizza, instant noodles, kimchi, red wine, and retort-pouch curry, with 5 replicates for all matrices. The spiking level was $0.1 \mathrm{mg} / \mathrm{kg}$, which was the same level as the evaluation level in the "Rapid analytical method for pesticides containing high levels in processed foods" (Office Memorandum, Standards and Evaluation Division, Department of Food Safety, Ministry of Health, Labour and Welfare, Japan, March 26, $2013)^{* 1}$. The results are shown in Table 2. The recoveries were in the range of $85-96 \%$ and the relative standard deviations (RSDs) were less than 7\%, which were within the acceptable range according to the criteria set by Japanese guidelines (Ministry of Health, Labour and Welfare, Tokyo, Japan, 2010). The matrix effects were evaluated by comparing the peak areas of matrixmatched standards with those of the standard in solvent (Table 3). As can be seen, the ratios were in the range of 1.01-1.10, indicating that no significant matrix effect was present. Fig. 2 shows representative chromatograms of a blank sample, a fortified sample, and a standard solution. No interfering peaks were found in the chromatograms of the blank samples at the retention time of tetramine, suggesting that the proposed method effectively removes co-extracted matrix components and has high selectivity. Based on a peak-to-peak signal-tonoise ratio of 3 , the limit of detection for tetramine was estimated to be $0.003 \mathrm{mg} / \mathrm{kg}$. 
Table 3. Matrix effects of tetramine in processed foods

\begin{tabular}{lc}
\hline \hline Sample & Matrix effect $^{\mathrm{a}}$ \\
\hline Broiled eel & 1.02 \\
Butter & 1.09 \\
Cheese & 1.01 \\
Chinese dumpling & 1.04 \\
Hamburger & 1.10 \\
Pizza & 1.06 \\
Instant noodles & 1.04 \\
Kimchi & 1.05 \\
Red wine & 1.04 \\
Retort-pouch curry & 1.02 \\
\hline
\end{tabular}

${ }^{\text {a }}$ Matrix effect $=$ peak area of matrix-matched standard/peak area of tetramine in pure solvent.

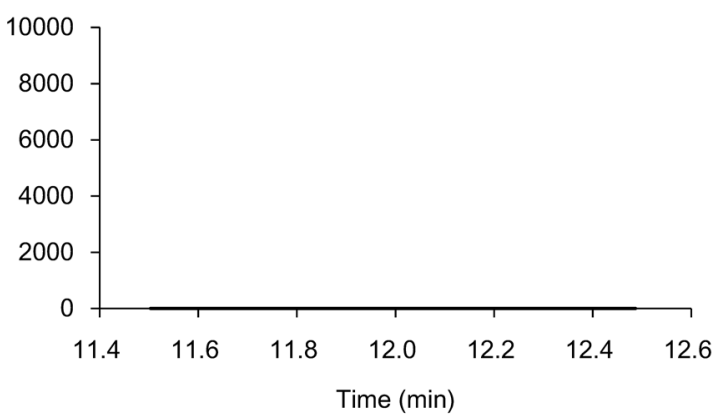

(b)

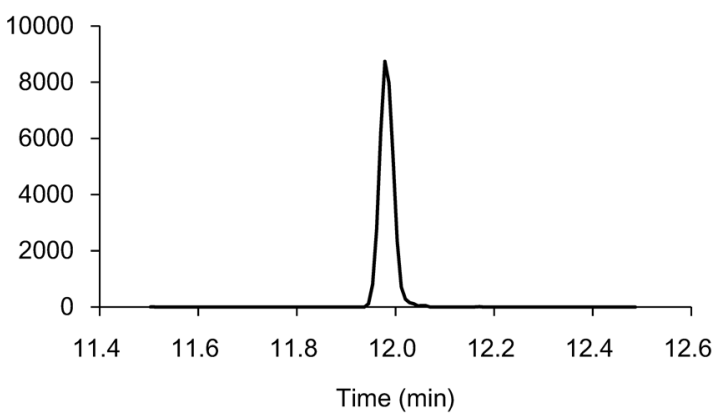

(c)

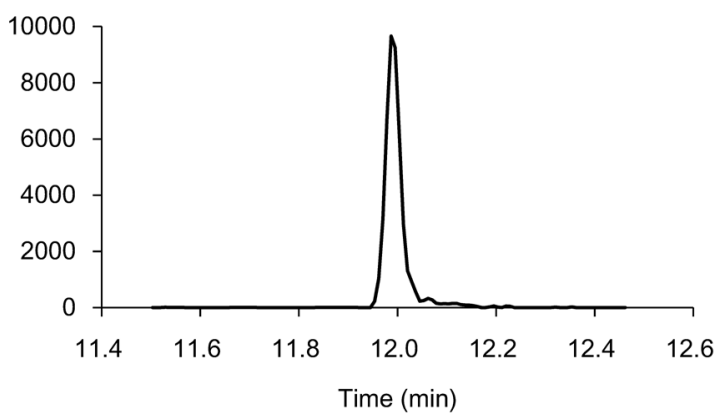

Fig. 2. Chromatogram of (a) extract of blank broiled eel, (b) extract of blank broiled eel spiked with $0.1 \mathrm{mg} /$ $\mathrm{kg}$ of tetramine, and (c) a $0.01 \mu \mathrm{g} / \mathrm{mL}$ standard solution of tetramine

\section{Conclusion}

In this study, we have developed a GC-MS/MS method for the determination of the highly toxic rodenticide, tetramine, in various processed foods. Acetonitrile/hexane partitioning and cleanup with a tandem graphitized carbon/PSA column effectively removed co-extractives without loss of the analyte. The recovery tests from 10 processed foods showed that the proposed method achieved good performance in terms of recovery and repeatability, and matrix effects were negligible. Therefore, the developed method is considered to be suitable for determining tetramine in processed foods.

\section{References}

1) Whitlow, K. S., Belson, M., Barrueto, F., Nelson, L., Henderson, A. K. Tetramethylenedisulfotetramine: Old agent and new terror. Ann. Emerg. Med., 45, 609-613 (2005).

2) Zhang, Y., Su, M., Tian, D. P. Tetramine poisoning: A case report and review of the literature. Forensic Sci. Int., 204, e24-e27 (2011).

3) Barrueto, F. Jr., Furdyna, P. M., Hoffman, R. S., Hoffman, R. J., Nelson, L. S. Status epilepticus from an illegally imported Chinese rodenticide: “Tetramine”. J. Toxicol. Clin. Toxicol., 41, 991-994 (2003).

4) Xu, X., Song, G., Zhu, Y., Zhang, J., Zhao, Y., Shen, H., Cai, Z., Han, J., Ren Y. Simultaneous determination of two acute poisoning rodenticides tetramine and fluoroacetamide with a coupled column in poisoning cases. J. Chromatogr. B, 876, 103-108 (2008).

5) Hamelin, E., Swaim, L., Thomas, J., Kobelski, R., Johnson, R. C. Quantitation of tetramethylenedisulfotetramine in human urine using isotope dilution gas chromatography mass spectrometry (GC/MS and GC/MS/MS). J. Chromatogr. B, 878, 2541-2547 (2010).

6) Luan, T., Li, G., Zhao, M., Zhang, Z. Rapid detection of tetramethylenedisulfotetramine in human blood by solidphase microextraction/gas chromatography. Anal. Chim. Acta, 404, 329-334 (2000).

7) De Jager, L. S., Perfetti, G. A., Diachenko G. W. Analysis of tetramethylenedisulfotetramine in foods using solid-phase microextraction-gas chromatography-mass spectrometry. J. Chromatogr. A, 1192, 36-40 (2008).

8) Owens J., Hok, S., Alcaraz, A., Koester, C. Quantitative analysis of tetramethylenedisulfotetramine (tetramine) spiked into beverages by liquid chromatography-tandem mass spectrometry with validation by gas chromatography-mass spectrometry. J. Agric. Food Chem., 57, 40584067 (2009). 